\title{
INEQUIDADES DE GÉNERO Y SU IMPACTO EN SALUD MATERNA Y PERINATAL. UNA REVISIÓN NARRATIVA
}

Gender Inequalities and their Impact on Maternal and Perinatal Health. A Narrative Review

Jovita Ortiz-Contreras ${ }^{1}$, Jael Quiroz-Carreño ${ }^{2}$, Maribel Mella-Guzmán ${ }^{3}$

Autor correspondencia: Jael Quiroz Carreño

Correo electrónico: jaelquirozc@uchile.cl

1. Matrona, Especialista en Perinatología. Magister en Salud Sexual y Reproductiva. Departamento de Promoción de la Salud de la Mujer y el Recién Nacido de la Facultad de Medicina de la Universidad de Chile. (Santiago, Chile)

2. Matrona, Magister en Salud Pública con especialización en Políticas Públicas y Economía de la Salud. Departamento de Promoción de la Salud de la Mujer y el Recién Nacido de la Facultad de Medicina de la Universidad de Chile. (Santiago, Chile)

3. Matrona, Magister en Bioética. Departamento de Promoción de la Salud de la Mujer y el Recién Nacido de la Facultad de Medicina de la Universidad de Chile. (Santiago, Chile)

Recibido: 30/08/2020 Aceptado: 02/02/2021

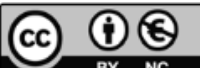




\section{RESUMEN}

OBJETIVO. Se pretende explorar el impacto de las inequidades de género en la salud sexual y reproductiva, con foco principal en la salud materna y perinatal. MÉTODO. Se realizó una revisión narrativa de categoría experto, orientada a la revisión temática con enfoque problematizador. Se llevó a cabo una búsqueda principal en las bases de datos Pubmed y Scielo, con una búsqueda secundaria en Google Scholar y adición por técnica de bola de nieve. Se llevó a cabo un análisis con relación a categorías de impacto, según dimensiones ex priori, y con base en un enfoque tipo hermenéutico interpretativo. RESULTADOS. Del análisis se desprendieron cuatro categorías que dan cuenta de respuestas generales al objetivo de la revisión: garantías de derechos reproductivos; discriminación nutricional de género e impacto materno-perinatal; subvaloración social explícita e impacto perinatal; y violencia de género y riesgo reproductivo y perinatal. CONCLUSIONES. La salud de las mujeres, así como su salud reproductiva, materna y perinatal, se encuentran permeadas por la condición de género, generando un impacto en el desarrollo pleno de los derechos sexuales y reproductivos, como al acceso a calidad de vida que propenda a mantener su potencial saludable. Por tanto, los indicadores que expresan la calidad de dicha salud requieren incorporación prioritaria de la transversalización de enfoque de género y derechos.

Palabras clave: Inequidad de género; Salud Materna y Perinatal; Mortalidad Materna; Salud Sexual y Reproductiva.

\section{ABSTRACT}

OBJECTIVE. This study aims to explore the impact of gender inequities on sexual and reproductive health, with a primary focus on maternal and perinatal health. METHOD. A narrative review of the expert category was carried out, aimed at a thematic review with a problematizing approach. A main search was carried out in the Pubmed and Scielo databases, with a secondary search in Google Scholar and addition by snowball technique. An analysis was carried out in relation to impact categories, according to ex priori dimensions, and based on an interpretive

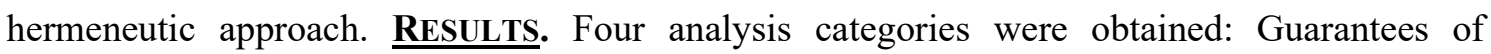
reproductive rights, nutritional gender discrimination and maternal-perinatal impact, explicit social undervaluation and perinatal impact, gender violence and reproductive and perinatal risk. CONCLUSIONS. The women's health and their reproductive, maternal and perinatal health, are permeated by gender, generating an impact on the full development of sexual and reproductive rights, as well as access to quality of life that tends to maintain their healthy potential. Therefore, the indicators that express the quality of said health require priority incorporation of gender and rights mainstreaming.

Keywords: Gender inequality; Maternal and Perinatal Health; Maternal Mortality; Sexual and Reproductive Health. 


\section{INTRODUCCIÓN}

El género es un constructo social multidimensional que involucra la asignación cultural de roles diferenciados a hombres y mujeres (OMS, 2020). Las desigualdades e inequidades de género, resultantes de dichos roles, así como de estereotipos basados en estos, implican muchas veces la vulneración de derechos humanos fundamentales, los cuales trascienden las diversas culturas.

La problemática asociada a estereotipos de género puede influir en aspectos tan fundamentales para el desarrollo humano como son el acceso a educación, empleo, nutrición y salud, así como también en el ejercicio de la autonomía y libertad de las mujeres. Estas consecuencias se traducen en una mayor vulnerabilidad en las diferentes etapas de la vida y, directa y/o indirectamente, a su descendencia (Brinda, Rajkumar y Enemark, 2015).

Informes respecto al desarrollo mundial señalan que la identificación de la igualdad de género es un instrumento para mejorar la productividad general de la economía de una sociedad, y que la desigualdad injusta en el acceso y distribución de recursos y oportunidades entre hombres y mujeres, traducida en inequidad de género, obstaculiza el crecimiento económico y el desarrollo social de los países (Banco Mundial, 2012). En respuesta a lo anterior, el Programa de Desarrollo de las Naciones Unidas (PNUD) propuso un Índice de Desigualdad de Género (IDG), el cual consiste en una medida compuesta que incluye tres dimensiones: salud reproductiva, empoderamiento, y participación laboral de las mujeres (PNUD, 2019). Estas dimensiones derivan de cinco aspectos de desigualdad: nivel educacional, representación parlamentaria, fuerza de la participación laboral, tasa de fecundidad adolescente y tasa de mortalidad materna y perinatal.

En consecuencia, se estima que la vulneración de cualquier elemento asociado a dichos componentes, principalmente los asociados a aspectos reproductivos, perpetúan las desigualdades e inequidades de género. Asimismo, se estiman consecuencias transgeneracionales aún en estudio, de carácter tanto biológico, social, económico y político para la sociedad (PNUD, 2019; Brinda, Rajkumar y Enemark, 2015). Estas consecuencias pueden complejizarse, toda vez que se potencian con otras vulnerabilidades y determinantes sociales, como son el bajo nivel socioeconómico, la baja 
tasa de escolaridad, etnia, contexto sociopolítico desfavorable y condición de migración, entre otras (Martins et al., 2013; Krieger et al., 2018).

En el contexto reproductivo, se ha demostrado que la maternidad derivada de una desigualdad biológica entre sexos constituye por sí misma un evento determinante en los proyectos de vida de las mujeres (Royo Prieto, 2013). Lo anterior se refleja como una fuente de inequidad, en la medida en que las sociedades condicionan dicha diferencia de disfrute de oportunidades entre hombres y mujeres, transformándose en claras inequidades de género (Hauser Dacer, 2017). Sin embargo, más allá de las inequidades propias de la maternidad estereotipada, se revela una problemática como máxima expresión de desigualdad: la mortalidad materna.

La mortalidad materna es un gran problema de salud pública y compromete aspectos importantes del género y los derechos, puesto que es afectada adicionalmente por todos los determinantes sociales de salud. En este sentido, debe ser visualizada como una problemática de alto impacto y trascendencia, ya que sus indicadores se comportan como una fiel representación del nivel de desarrollo de un país (Banco Mundial, 2012; Jahan, 2016; Brinda, Rajkumar y Enemark, 2015).

Según lo establecido en la agenda de los Objetivos de Desarrollo Sostenible (ODS) de las Naciones Unidas, dicha mortalidad sigue siendo un reto importante, especialmente en países de bajo índice de desarrollo humano y bajo IDG. Frente a esta preocupación constante, se han planteado los objetivos 3 y 5 , que buscan garantizar una vida sana, promover el bienestar en todas las edades y lograr la igualdad de género poniendo fin a todas las formas de discriminación contra las mujeres y niñas (Naciones Unidas, 2015; Naciones Unidas, 2018). Un claro ejemplo de esta realidad es que, en los países clasificados como Estados frágiles, con bajos índices de desarrollo humano y de IDG, el riesgo de morir asociado a la gestación es 99 veces mayor que en países de alto desarrollo humano. Esto demuestra que las condiciones de vulnerabilidad se dan en un escenario de descomposición de los sistemas de salud e inequidad, la cual estaría asociada a la condición reproductiva de las mujeres, permeadas a su vez por la construcción cultural de género en el país de origen (Grupo Interagencial de las Naciones Unidas, 2008; OMS, 2019; Menéndez y Lucas, 2016).

Adicionalmente, más allá de la mortalidad materna como indicador puro, subyacen problemáticas complejas de inequidades de género en el contexto reproductivo, las cuales 
se encuentran invisibilizadas y subvaloradas en el abordaje multidimensional del problema. Es por ello por lo que, ante este escenario adverso, cabe la necesidad de generar e identificar evidencia que visualice los caminos y consecuencias de dichas inequidades, principalmente las que afectan el goce y disfrute de derechos sexuales y reproductivos de las mujeres. El presente artículo pretende explorar el impacto de las inequidades de género en la salud reproductiva, con foco principal en la salud materna y perinatal.

\section{MÉTODO}

Se realizó una revisión narrativa de categoría experto (González-Garay, Mayorga-Butrón y Ochoa-Carrillo, 2015), orientada a la revisión temática con enfoque problematizador, que busca una reflexión en torno a la pregunta planteada en base a la literatura seleccionada. Se desarrolló una búsqueda inicial en inglés y español en las bases de datos Pubmed y Scielo, respectivamente. Estas bases fueron seleccionadas por contener cada una la mayor representatividad y heterogeneidad de publicaciones en los idiomas respectivos. Para la búsqueda de los artículos se utilizaron las palabras claves: «gender inequality index», «maternal health» $\mathrm{y}$ «reproductive health». Además, se realizó una búsqueda secundaria en Google Scholar controlando el sesgo de publicación. Para el buscador mencionado, se seleccionaron las dos primeras páginas, ya que posterior a esto, los resultados eran redundantes y escapaban de los objetivos de la revisión (Pham et al., 2014).

De forma paralela, a la selección de la literatura identificada, se aplicó una estrategia de incorporación de artículos por bola de nieve, donde se agregó de forma manual todo aquel estudio y/o documento que respondiera a nuestro interrogante y que haya sido citado en los estudios inicialmente seleccionados.

Se limitó la búsqueda a los años 2010 y 2020, abarcando publicaciones que dieran cuenta de la temática. Los criterios de inclusión fueron: estudios que se refirieran a la atención de mujeres en el contexto de salud reproductiva y/o salud materna. Se excluyeron estudios moleculares, en animales, guías clínicas, estudios de caso, de costo y caso clínico. 
Todas las citas se importaron al administrador bibliográfico Mendeley (versión 1.19.5) y las citas duplicadas se eliminaron manualmente. Al realizar la búsqueda inicial según palabras claves, se encontró un total de 103 estudios que, tras la lectura de título y resumen, y al aplicar los criterios de inclusión y exclusión, se redujo a un total de 41 artículos, incluidos aquellos recolectados por bola de nieve. Finalmente, en el análisis de relevancia, se seleccionaron 37 referencias para su lectura completa, para una posterior triangulación de lectura por parte de las autoras. Este proceso facilitó la resolución de conflictos de selección, y garantizó la coherencia entre revisores y la extracción de datos que dan respuesta a los objetivos de la investigación (Figura 1).

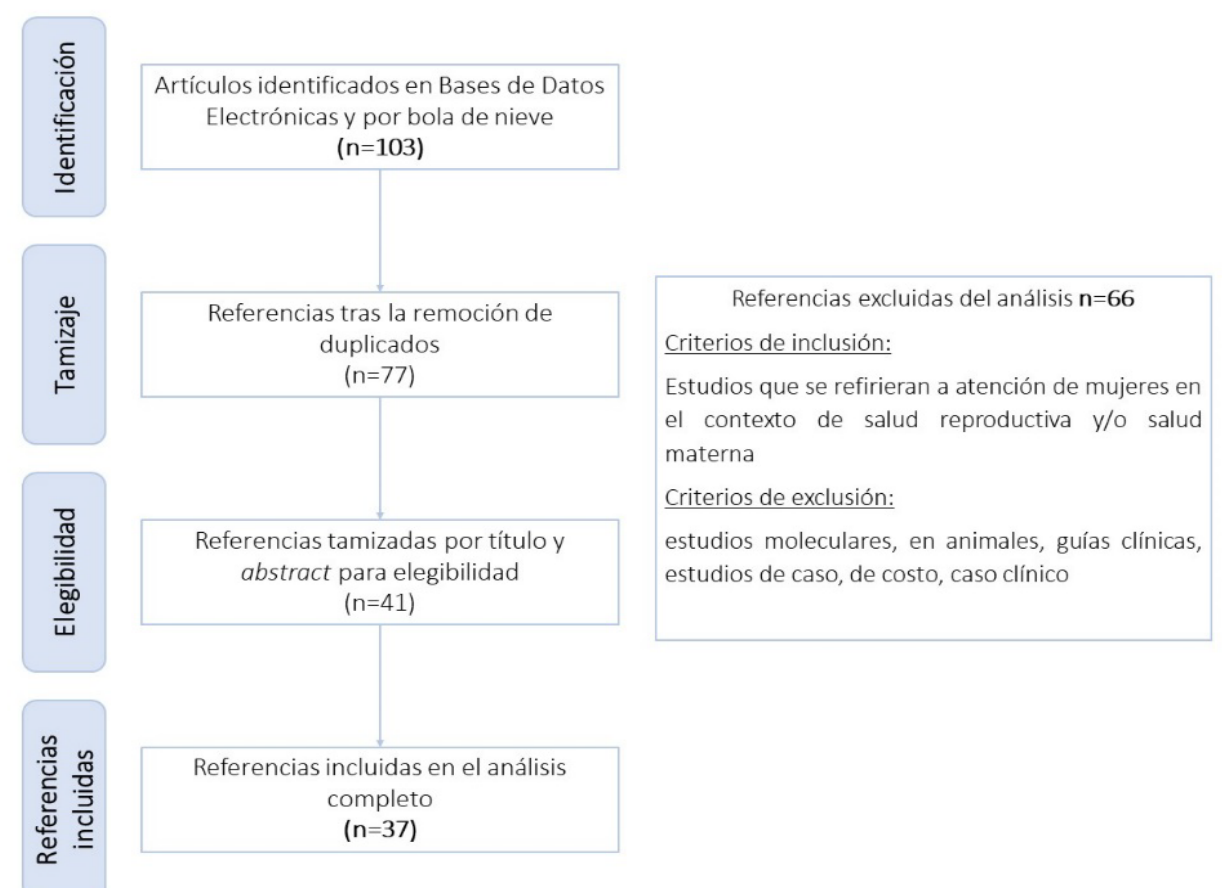

Figura 1. Diagrama de flujo tipo PRISMA modificado para revisión narrativa

Fuente: Elaboración propia en base a PRISMA modificado para Scoping Review

Por su parte, el análisis se realizó con relación a categorías de impacto identificadas según la pregunta de investigación, en dimensiones ex priori, y con base en un enfoque tipo hermenéutico-interpretativo. Este análisis combina la evidencia emanada de la búsqueda con la interpretación de las autoras (Neubauer, Witkop y Varpio, 2019).

\section{RESULTADOS Y DISCUSIÓN}

De los 37 artículos seleccionados, dos correspondían a revisiones de literatura de los años 2011 y 2016. Cinco estudios fueron incluidos en la revisión de bola de nieve, aunque su 
publicación fuera previa a 2010, por la relevancia del estudio. Se incluyeron cuatro declaraciones y/o propuestas de la Organización Mundial de la Salud respecto al tema.

En general, en los documentos se consideraron como unidad de análisis las mujeres y su relación con los sistemas de salud. La mayoría de los estudios tuvo por objetivo determinar la asociación de las inequidades de género con la salud materna. Del análisis se desprendieron cuatro categorías que dan cuenta de respuestas generales a la pregunta de la revisión. Estas fueron: (1) garantías de derechos reproductivos; (2) discriminación nutricional de género e impacto materno-perinatal; (3) subvaloración social explícita e impacto perinatal; (4) violencia de género y riesgo reproductivo y perinatal.

\section{Garantías de derechos reproductivos}

Los derechos reproductivos, genuinamente orientados a proteger la libertad y autonomía de todas las personas para decidir con responsabilidad, denotan aspectos como la decisión de cuántos, en qué momento y con quién se desea gestar hijos e hijas, y poseen una clara imparcialidad de género en su vivencia (Robinson et al., 2017).

Ante este hecho, históricamente se ha otorgado el «trabajo reproductivo» a las mujeres, con consecuencias concomitantes en diversos aspectos de su vida y su salud (Hauser Dacer, 2017; Kostov, Koppula y Babenko, 2018). Es por ello por lo que existen países donde los estereotipos de género impactan explícitamente en la autonomía personal y económica de las mujeres, impidiendo que tengan acceso libre y oportuno a los servicios de salud (McDougal et al., 2017; Kostov, Koppula y Babenko, 2018). Asimismo, a pesar de las múltiples declaraciones internacionales tendientes a fortalecer el respeto y la no discriminación de niñas y mujeres, aún existen países en los que se justifica y permite el matrimonio infantil y la mutilación genital femenina, entre otros tipos de violaciones de derechos humanos, y en los cuales aún no se han planteado políticas sanitarias tendientes a la protección y mejora efectiva del cuidado y asistencia de la gestación, parto y puerperio (Grupo Interagencial de las Naciones Unidas, 2008).

Por su parte, el matrimonio infantil, así como adolescente, afecta desproporcionadamente más a mujeres que a hombres, exponiendo una clara vulneración de libertad y autonomía. Este fenómeno, guarda relación directa con un mayor riesgo de gestaciones no deseadas, infecciones de transmisión sexual (ITS), infección por VIH/SIDA, morbilidad y mortalidad maternas y de los hijos (McDougal et al., 2017; Adeyinka, Olakunde y Muhajarine, 2019; Banda et al., 2017). A su vez, el embarazo en 
la niñez y adolescencia se asocia a mayor riesgo de morbilidades y a graves complicaciones (físicas, psicológicas y sociales) que requieren de mayor tiempo para su recuperación, afectando la economía de sus familias y comunidades (Decker et al., 2017; McDougal et al., 2017). Tanto es así que el impacto de este en salud materna y perinatal trasciende lo social, económico y político, ya que perpetúa círculos de pobreza, estigmatización, discriminación $\mathrm{y}$ brechas de disfrute de derechos humanos fundamentales (Arora, 2012).

En este escenario, se ha demostrado que la vulneración de la salud de la mujer, y específicamente la genuina vulneración de sus derechos sexuales y derechos reproductivos, se relaciona directamente con mayor morbimortalidad materna y con mayor riesgo de morir por esta causa (PNUD, 2019; Grupo Interagencial de las Naciones Unidas, 2008). En este sentido, los hallazgos sugieren que no solo se debe trabajar en la disminución de brechas en salud, sino en potenciar la disminución de brechas de género educativas y económicas para conducir a mejoras en la salud sexual, reproductiva y materna en países con ingresos bajos y medios (Choe, Cho y Kim, 2017).

Por otra parte, y asociado al disfrute de la sexualidad y la reproducción segura y por elección, la regulación de la fecundidad se ha enfocado mayormente en la producción de anticoncepción femenina. Este fenómeno denota, evidentemente, un sesgo de género importante, que posiciona socialmente a las mujeres como responsables absolutas de lo reproductivo (De Looze et al., 2019; Quintana Llanio, 2017). En este sentido, el concepto de «demanda insatisfecha de planificación familiar» alude a la brecha entre las intenciones reproductivas de la mujer y su comportamiento anticonceptivo. Este monitoreo para los ODS se expresa como un porcentaje basado en las mujeres que están casadas o en unión consensual, lo cual plantea un análisis reduccionista en cuanto a la producción y efectos multidimensionales de la problemática (Consejo de Derechos Humanos, 2019; Díez Peralta, 2019). A su vez, los países en los cuales se reporta mayor demanda insatisfecha se relacionan directamente con mayores tasas de mortalidad materna y perinatal (Gil-González et al., 2008; Gaye et al., 2010).

En consecuencia, la falta de garantías para la vivencia plena de la sexualidad, permeada por problemáticas de educación sexual, demandas insatisfechas de métodos anticonceptivos y la ausencia de coberturas de abortos legales y seguros influyen en la autonomía de decisión respecto a una concepción deseada. Lo anterior expone a las mujeres a una suerte de «esclavitud reproductiva», traducida en una franca vulneración 
de sus derechos sexuales y reproductivos. Este fenómeno expone exclusivamente a las mujeres a un mayor riesgo de mortalidad materna, estigmatización social e inequidades de género futuras, reiniciando un ciclo de vulneración asociada al género (Marphatia et al., 2016; Brinda, Rajkumar y Enemark, 2015).

\section{Discriminación nutricional de género e impacto perinatal}

La desigualdad de género, que se encuentra detrás de factores de riesgo biopsicosociales en el contexto reproductivo, como la malnutrición materna, repercute directamente en la gestación. Es posible observar consecuencias a corto y largo plazo sobre las niñas y niños que son producto de dichas gestaciones, lo cual implica un círculo de inequidad que no solo afecta el derecho de las mujeres, sino también el de las generaciones venideras, independiente del género (Osmani y Sen, 2003).

Se ha señalado, por ejemplo, que una de las principales causas de la malnutrición materna en países en desarrollo responde a razones sociales como la discriminación de género. Esto, bajo la concepción cultural de que los alimentos se deben disponer prioritariamente para hombres, conllevaría una suplementación nutricional inadecuada para el género femenino. A su vez, las consecuencias biológicas asociadas a la subalimentación se traducen en mayor riesgo de infecciones maternas y de patologías perinatales, como prematuridad, restricción del crecimiento fetal y bajo peso al nacer (Sharma, Shastri y Sharma, 2016; Brinda, Rajkumar y Enemark, 2015), las cuales se ha demostrado que pueden desencadenar mayor mortalidad y morbilidad fetal y neonatal, así como alteraciones del crecimiento y desarrollo neuroconductual y enfermedades crónicas en niños y niñas (Osmani y Sen, 2003; Sharma, Shastri y Sharma, 2016).

Es así, que se demostró que las mujeres de países de ingresos bajos y medios poseen mayor desigualdad e inequidad de género, revelando una significativa asociación entre el índice de desigualdad de género y mortalidad neonatal e infantil (Bhalotra y Rawlings, 2011; Brinda, Rajkumar y Enemark, 2015). Asimismo, Brinda (2015) sugiere «que las iniciativas para reducir las tasas de mortalidad materna e infantil debería extenderse más allá de las intervenciones médicas y deben dar prioridad a los derechos y la autonomía de la mujer». 


\section{Subvaloración social explícita e impacto perinatal}

Si bien muchos países han realizado al menos declaraciones de intención respecto a avanzar en inequidades de género, la problemática de países del Oriente y Oriente Medio, asociada a la subvaloración femenina socialmente explícita desde el nacimiento, produce una discriminación de género que desarrolla un impacto perinatal. Este impacto perinatal se ha demostrado en un mayor riesgo de infanticidio y de abandono de recién nacidas de sexo femenino, las cuales poseen significativamente menos probabilidades de sobrevivir que los niños de sexo masculino (Nielsen et al., 1997; Diamond-Smith, Luke y McGarvey, 2008; Brinda, Rajkumar y Enemark, 2015).

\section{Violencia de género y riesgo reproductivo y perinatal}

La violencia de género está particularmente presente durante la gestación y el posparto como otro factor de riesgo reproductivo ligado al género. En algunos países, los niveles de embarazo no deseado son de dos a tres veces mayores en las mujeres víctimas de violencia en manos de su pareja que en aquellas que no la sufren. Entre el $3 \%$ y el $44 \%$ de las embarazadas en América Latina y el Caribe informan haber sufrido actos de violencia durante el embarazo, estimándose su prevalencia entre 1,2 \% a un 27,6 \% (Bott et al., 2014).

Se ha asociado la presencia de violencia no solo a una alta generación de estrés en mujeres gestantes, sino que también a gestaciones a edades más precoces, y también a mayor riesgo perinatal, con la consecuente generación de parto pretérmino, bajo peso al nacimiento, muerte fetal o neonatal, muerte en el primer año de vida, y mayor morbilidad respiratoria (James, Brody y Hamilton, 2013; Decker et al., 2017). Asimismo, los hijos de madres víctimas de violencia, durante el primer año de vida, presentan mayor riesgo de mortalidad infantil y menor adhesión a controles de salud (Donovan et al., 2016).

Por su parte, la violencia de género aumenta el riesgo de las mujeres a adquirir la infección por VIH y otras enfermedades de transmisión sexual durante la gestación (Blas y Sivasankara Kurup, 2010; Jewkes et al., 2010). En este sentido, la falta de autonomía debido a este contexto obstaculiza el acceso a la educación sanitaria y preventiva de manera equitativa, así como a los servicios de salud para la prevención de transmisión vertical, tanto de VIH como de ITS (Kamiya, 2011; OMS, 2009). Asimismo, se ha estudiado el impacto de la violencia doméstica contra la mujer, con resultados claros que afectan el crecimiento y nutrición del niño o niña, durante la gestación hasta los 36 
primeros meses de vida, teniendo consecuencias no solo en la madre, sino también en los/las hijos/as (Yount, DiGirolamo y Ramakrishnan, 2011; Sethuraman, Lansdown y Sullivan, 2006).

De forma adicional, existe un tipo de violencia específica hacia la mujer, en contextos en que debiese considerarse protegida y cuidada, como es el caso de la violencia institucionalizada. Este tipo de violencia ha sido reconocida por la Organización Mundial de la Salud (OMS) en múltiples ocasiones, con documentos en los que declara su preocupación inherente (2014). La falta de respeto y maltrato durante la atención en salud de la mujer se proyecta incluso en los partos de mujeres de bajo riesgo y es, en definitiva, una forma de ejercer «violencia de género simbólica y epistémica», que se ve naturalizada en las inequidades de poder (OMS, 2015; Bellón Sánchez, 2015). En este contexto, la OMS ha llamado a prevenir y eliminar la falta de respeto durante el proceso reproductivo y nacimiento, entendiendo que dicha situación tiene consecuencias tanto físicas como emocionales que atentan contra toda autonomía y derecho de mujeres, niñas y niños en el mundo, así como también al potencial de salud de su proceso reproductivo e indirectamente a la salud de su descendencia (Dahlen et al., 2013; 2016).

En concordancia con lo anterior, la forma más reconocida de violencia institucional es la violencia obstétrica, que se ha sindicado como una de las principales formas de vulneración a mujeres, asociada al género durante su proceso reproductivo. Dicha violencia refleja modelos de patriarcado en la asistencia del proceso reproductivo y una de las mayores desigualdades de género en la atención de salud de las mujeres (Al Adib Mendiri et al., 2017; Solnes Miltenburg et al., 2018; Bellón Sánchez, 2015). Adicionalmente, existen hipótesis que defienden que el estrés producido por ambientes violentos en contextos de parto puede desregular la fisiología femenina del nacimiento, con potencial impacto en la salud materna y de los individuos a largo y mediano plazo (Dahlen et al., 2013), afectando directa e indirectamente sus derechos a una vida saludable.

\section{LIMITACIONES DEL ESTUDIO}

Por tratarse de una revisión narrativa de categoría experto, las limitaciones de este estudio corresponden esencialmente a las propias de la metodología de dicha revisión, esto es, que los elementos concluidos se basan principalmente, como su nombre indica, en la 
opinión de experto con base en evidencia científica, más que en un proceso de sistematización de esta. Adicionalmente, la escasa evidencia de desigualdades de género en países de mayor índice de desigualdad puede haber generado omisión de otras dimensiones de impacto. Sin embargo, el tipo de análisis realizado permitió complementar los hallazgos con la experiencia de las autoras en la respuesta a la pregunta de estudio.

\section{CONCLUSIONES}

La salud general de las mujeres, así como su salud reproductiva, materna y perinatal, se encuentra claramente permeada por la condición de género establecida como constructo social, cuyo impacto se manifiesta en diversos ámbitos generados por estereotipos de género, violencia y desigualdades sociales. De este modo, las demandas de derechos humanos de las mujeres deben ser articuladas desde un enfoque que tenga el género como protagonista, teniendo en cuenta que las desigualdades económicas, sanitarias y educacionales confabulan negativamente en contra de estas y su descendencia. Es por esto por lo que los indicadores que expresan la calidad de vida y salud, considerados como factores involucrados en el desarrollo de los países, requieren incorporación prioritaria de dicho enfoque, como una herramienta fundamental, multifactorial y multidimensional en su abordaje.

En este sentido, es posible concluir que la literatura muestra diversas asociaciones entre la desigualdad de género y resultados maternos y perinatales, así como también resultados transversales en salud. Por tanto, para lograr no solo los ODS, sino también la autonomía de las mujeres y un impacto real de las políticas en la mejora de la salud, se requiere urgentemente la asimilación de la transversalización de género, perspectiva de derechos, curso de vida y de determinantes sociales.

La eliminación de desigualdades injustas debe ser una de las estrategias a potenciar en los años venideros con propuestas que sean prioritarias en cualquier contexto, incluyendo las instancias de crisis sociales, sanitarias y/o humanitarias, favoreciendo con ello no solo los resultados en salud de las mujeres y de las futuras generaciones, sino también el empoderamiento y autonomía de ellas y su aporte sustancial al desarrollo integral de las sociedades. 


\section{BIBLIOGRAFÍA}

Adeyinka, D. A.; Olakunde, B. O.; Muhajarine, N. Evidence of health inequity in child survival: spatial and Bayesian network analyses of stillbirth rates in 194 countries. En: Scientific Reports. 2019, vol. 9, núm. 1, pp. 1-11.

Al AdiB MendiRi, M.; et al. La violencia obstétrica: un fenómeno vinculado a la violación de los derechos elementales de la mujer. En: Medicina Legal de Costa Rica. 2017, vol. 34, núm. 1, pp. 104-111.

ARORA, R. Gender inequiality, economic development, and globalization: A state level analysis of India. En: The Journal of Developing Areas. 2012, vol. 46, núm. 1, pp. 147-164.

Banco Mundial. World Development Report 2012. Overview: Gender equality and development. BM: 2012.

BANDA, P. C., et al. Women at risk: Gender inequality and maternal health. En: Women and Health. 2017, vol. 57, núm. 4, pp. 405-429.

BELLÓN SÁNCHEZ, S. La violencia obstétrica desde los aportes de la crítica feminista y la biopolítica. En: Dilemata. 2015, núm. 18, pp. 93-111.

Bhalotra, S.; Rawlings, S. B. Intergenerational persistence in health in developing countries: The penalty of gender inequality? En: Journal of Public Economics. 2011, vol. 95, núms. 3-4, pp. 286-299.

Blas, E.; Sivasankara Kurup, A. Equity, social determinants and public health programmes. Ginebra: OMS, 2010.

BotT, S., et al. Violencia contra las mujeres en América Latina y el Caribe: Análisis comparativo de datos poblacionales de 12 países. Washington: OPS, 2014.

Brinda, E. M.; RAJKumar, A. P.; ENEMARK, U. Association between gender inequality index and child mortality rates: A cross-national study of 138 countries. En: BMC Public Health. 2015, vol. 15, núm. 1, pp. 3-8.

Choe, S.-A. A.; CHO, S. Il; KIM, H. Gender gap matters in maternal mortality in low and lower-middle-income countries: A study of the global Gender Gap Index. Global En: Public Health. 2017, vol. 12, núm. 9, pp. 1065-1076.

Consejo de Derechos Humanos. Matrimonio infantil, precoz y forzado en situaciones humanitarias. Asamblea General Naciones Unidad, vol. 41, 2019.

DAHLEN, H. G., et al. Childbirth and consequent atopic disease: Emerging evidence on epigenetic effects based on the hygiene and EPIIC hypotheses. En: BMC Pregnancy and Childbirth. 2016, vol. 16, núm. 1, pp. 1-8.

DAHLEN, H. G., et al. The EPIIC hypothesis: intrapartum effects on the neonatal epigenome and consequent health outcomes. En: Medical hypotheses. 2013, vol. 80, núm. 5, pp. 656-662. 
De LoOze, M., et al. Country-Level Gender Equality and Adolescents' Contraceptive Use in Europe, Canada and Israel: Findings from 33 Countries. En: Perspectives on Sexual and Reproductive Health. 2019, vol. 51, núm. 1, pp. 43-53.

DECKER, M. R., et al. Early adolescent childbearing in low- and middle-income countries: Associations with income inequity, human development and gender equality. En: Health Policy and Planning. 2017, vol. 32, núm. 2, pp. 277-282.

DiAmond-SMith, N.; LUKE, N.; MCGARVEY, S. «Too many girls, too much dowry»: Son preference and daughter aversion in rural Tamil Nadu, India. En: Culture, Health and Sexuality. 2008, vol. 10, núm. 7, pp. 697-708.

DíEz PERAlTA, EVA. El matrimonio infantil y forzado en el Derecho internacional: Un enfoque de género y de derechos humanos. Valencia: Tirant lo Blanch, 2019

DonOVAn, B. M., et al. Intimate partner violence during pregnancy and the risk for adverse infant outcomes: a systematic review and meta-analysis. Londres: Blackwell, 2016.

GAYe, A., et al. Human Development Research Paper 2017/46. Measuring Key Disparities in Human Development: The Gender Inequality Index. United Nations Development Programme, 2010. Disponible en: http:/hdr.undp.org/en/content/measuring-key-disparities-human-development

GIL-GonZÁlez, D., et al. Valoración de los objetivos de desarrollo del milenio mediante la revisión de la literatura científica en 2008. En: Revista Espanola de Salud Pública. 2008, vol. 82, núm. 5, pp. 455-466.

GONZÁlez-GARAY, A. G.; MAYORGA-ButróN, J. L.; OCHOA-CARRILlO, F. J. Revisiones sistemáticas de la literatura, la piedra angular de la medicina basada en evidencia. Documento de la serie Medicina basada en evidencia, 2 de 3. En: Gaceta Mexicana de Oncología. 2015, vol. 14, núm. 2, pp. 103-106.

GRUPo InTERAGENCIAL De LAS NACIONES UnidAs. Género y los Objetivos de Desarrollo del Milenio, Mortalidad materna, género y desarrollo. México: ONU, 2008

HAUSER DACER, J. Embarazo y Maternidad, las Desigualdades de Género y los Aportes del Arteterapia. En: Arteterapia. Papeles de arteterapia y educación artística para la inclusión social. 2017, vol. 11, pp. 151-161.

JAHAN, S. Overview: Human Development Report 2016. Human Development for Everyone. New York: 2016. Disponible en: http://hdr.undp.org/en/2016-report

JAmeS, L.; Brody, D.; Hamilton, Z. Risk factors for domestic violence during pregnancy: A meta-analytic review. En: Violence and Victim. 2013, vol. 28, núm. 3, pp. 359-380.

JEWKES, R. K., et al. Intimate partner violence, relationship power inequity, and incidence of HIV infection in young women in South Africa: A cohort study. En: The Lancet. 2010, vol. 376, núm. 9734, pp. 41-48. 
KAMIYA, Y. Women's autonomy and reproductive health care utilisation: Empirical evidence from Tajikistan. En: Health Policy. 2011, vol. 102, núm. 2-3, pp. 304-313.

Kostov, S.; Koppula, S.; BABenKO, O. Gender differences in women's health and maternity care training: A scoping review. En: MedEdPublish. 2018, vol. 7, núm. 1, pp. 1-9.

KRIEGER, N., et al. Severe sociopolitical stressors and preterm births in New York City: 1 September 2015 to 31 August 2017. En: Journal of Epidemiology and Community Health. 2018, vol. 72, núm. 12, pp. 1147-1152.

Marphatia, A. A., et al. Associations of gender inequality with child malnutrition and mortality across 96 countries. En: Global Health, Epidemiology and Genomics. 2016, vol. 1.

Martins, E .F., et al. Perinatal mortality and socio-spatial inequalities. En: Revista Latino-Americana de Enfermagem. 2013, vol. 21, núm. 5, pp. 1062-1070.

MCDOUGAL, L., et al. Making the continuum of care work for mothers and infants: Does gender equity matter? Findings from a quasi-experimental study in Bihar, India. En: PLoS ONE. 2017, vol. 12, núm. 2, pp. 1-19.

MenÉndeZ, C. y LuCAS, A. Analizando la mortalidad materna desde un enfoque de equidad: la importancia de contar con datos de calidad. Barcelona: Institutio de Salud Global, 2016

ORgANIZACIÓN DE LAS NACIONES UNIDAS. Objetivos de desarrollo sostenible. Objetivo 3, Salud y Bienestar. Ginebra: ONU, 2015.

Neubauer, B. E.; WitKoP, C. T.; VARPIO, L. How phenomenology can help us learn from the experiences of others. En: Perspectives on Medical Education. 2019, vol. 8, núm. 2, pp. 90-97.

NIELSEN, B. B., et al. Reproductive pattern, perinatal mortality and sex preference in rural Tamil Nadu, South India: Community based, cross sectional study. En: British Medical Journal. 1997, vol. 314, núm. 7093, pp. 1521-1524.

Organización Mundial DE LA SALUd. Health and Women: today's evidence tomorrow's agenda. Ginebra: OMS, 2009.

ORganización Mundial De LA SALUD. Prevención y erradicación de la falta de respeto y el maltrato durante la atención del parto en centros de salud Declaración de la OMS. Ginebra: OMS, 2014. Disponible en:

https://www.who.int/reproductivehealth/topics/maternal perinatal/statementchildbirth/es/

ORGANIZACIÓN MUNDIAL DE LA SALUD. The prevention and elimination of disrespect and abuse during facility-based childbirth. Ginebra: OMS, 2015.

ORGANIZACIÓN DE LAS NACIONES UNIDAS. La Agenda 2030 y los Objetivos de Desarrollo Sostenible: una oportunidad para América Latina y el Caribe. Santiago: ONU, 2018. Disponible en: 
https://repositorio.cepal.org/bitstream/handle/11362/40155/24/S1801141_es.pdf.

Organizacion Mundial De La Salud. Mortalidad materna. Ginebra: OMS, 2019.

Disponible en: https://www.who.int/es/news-room/fact-sheets/detail/maternalmortality

Organización Mundial de la Salud. Género. Ginebra: OMS, 2020. Disponible en: https://www.who.int/es/news-room/fact-sheets/detail/gender

Osmani, S.; SEN, A. The hidden penalties of gender inequality: Fetal origins of ill-health. En: Economics and Human Biology. 2003, vol. 1, núm. 1, pp. 105-121.

PHAM, M. T., et al. 2014. A scoping review of scoping reviews: Advancing the approach and enhancing the consistency. En: Research Synthesis Methods. 2014, vol. 5, núm. 4, pp. 371-385.

Programa de Desarrollo de las Naciones Unidas (UNDP). Human Development Report: Gender Inequality Index (GII). UNDP, 2019. Disponible en:

http://hdr.undp.org/en/content/gender-inequality-index-gii

QUiNTANA LLANIO, L. Cuba: fecundidad y toma de decisions en torno a la reproducción. Miradas en contexto. En: Revista Novedades en Población. 2017, vol. 13, núm. 25 , pp. 110-129.

RoBINSON, J. L., et al. Interventions to address unequal gender and power relations and improve self-efficacy and empowerment for sexual and reproductive health decisionmaking for women living with HIV: A systematic review. En: PLOS ONE. 2017, vol. 12, núm. 8, pp. 1-19.

RoYo PRIETO, R. Maternidad, paternidad y desigualdad de género: los dilemas de la conciliación. En: Zerbitzuan. 2013, núm. 53, pp. 123-134.

SeTHURAMAN, K.; LANSDOWn, R.; Sullivan, K. Women's empowerment and domestic violence: The role of sociocultural determinants in maternal and child undernutrition in tribal and rural communities in South India. En: Food and Nutrition Bulletin. 2006, vol. 27, núm. 2, pp. 128-143.

Sharma, D.; Shastri, S.; Sharma, P. Intrauterine Growth Restriction: Antenatal and Postnatal Aspects. En: Clinical Medicine Insights: Pediatrics. 2016, vol. 10.

Solnes Miltenburg, A., et al. Disrespect and abuse in maternity care: individual consequences of structural violence. En: Reproductive Health Matters. 2018, vol. 26, núm. 53, pp. 88-106.

Yount, K. M.; Digirolamo, A. M.; RAMAKRISHNAN, U. Impacts of domestic violence on child growth and nutrition: A conceptual review of the pathways of influence. En: Social Science and Medicine. 2011, vol. 72, núm. 9, pp. 1534-1554. 